\title{
Comparison of diagnostic methods for the detection and quantification of the four sympatric Plasmodium species in field samples from Papua New Guinea
}

\author{
Anna Rosanas-Urgell ${ }^{1,2^{*}}$, Dania Mueller ${ }^{3,4}$, Inoni Betuela ${ }^{1}$, Céline Barnadas ${ }^{1}$, Jonah Iga ${ }^{1}$, Peter A Zimmerman5,
} Hernando A del Portillo ${ }^{2,6}$, Peter Siba ${ }^{1}$, Ivo Mueller ${ }^{1}$, Ingrid Felger ${ }^{3,4}$

\begin{abstract}
Background: Accurate diagnosis of Plasmodium infections is essential for malaria morbidity and mortality reduction in tropical areas. Despite great advantages of light microscopy (LM) for malaria diagnosis, its limited sensitivity is a critical shortfall for epidemiological studies. Robust molecular diagnostics tools are thus needed.

Methods: The present study describes the development of a duplex quantitative real time PCR (qPCR) assay, which specifically detects and quantifies the four human Plasmodium species. Performance of this method was compared to PCR-ligase detection reaction-fluorescent microsphere assay (PCR_LDR_FMA), nested PCR (nPCR) and LM, using field samples collected from 452 children one to five years of age from the Sepik area in Papua New Guinea. Agreement between diagnostic methods was calcualted using kappa statistics.

Results: The agreement of QPCR with other molecular diagnostic methods was substantial for the detection of P. falciparum, but was moderate for the detection of P. vivax, P. malariae and P. ovale. P. falciparum and P. vivax prevalence by qPCR was $40.9 \%$ and $65.7 \%$ respectively. This compares to $43.8 \%$ and $73.2 \%$ by $\mathrm{nPCR}$ and $47.1 \%$ and $67.5 \%$ by PCR_LDR_FMA. P. malariae and P. ovale prevalence was $4.7 \%$ and $7.3 \%$ by qPCR, $3.3 \%$ and $3.8 \%$ by nPCR, and $7.7 \%$ and $4.4 \%$ by PCR_LDR_FMA. Prevalence by LM was lower for all four species, being $25.4 \%$ for $P$. falciparum, $54.9 \%$ for $P$. vivax, $2.4 \%$ for $P$. malariae and $0.0 \%$ for $P$. ovale. The quantification by qPCR closely correlated with microscopic quantification for $P$. falciparum and $P$. vivax samples $(R 2=0.825$ and $R 2=0.505$, respectively). The low prevalence of $P$. malariae and $P$. ovale did not permit a solid comparative analysis of quantification for these species.

Conclusions: The GPCR assay developed proved optimal for detection of all four Plasmodium species. Densities by $L M$ were well reflected in quantification results by $q P C R$, whereby congruence was better for $P$. falciparum than for $P$. vivax. This likely is a consequence of the generally lower $P$. vivax densities. Easy performance of the qPCR assay, a less laborious workflow and reduced risk of contamination, together with reduced costs per sample through reduced reaction volume, opens the possibility to implement qPCR in endemic settings as a suitable diagnostic tool for large epidemiological studies.
\end{abstract}

\section{Background}

Rapid and accurate diagnosis of Plasmodium infections is crucial for morbidity and mortality reduction in tropical areas, specially in regions where mixed infections are prevalent such as Papua New Guinea (PNG), where all four

\footnotetext{
* Correspondence: anna.rosanas@pngimr.org.pg

${ }^{1}$ Papua New Guinea Insitute of Medical Research, Madang 511, Papua New Guinea

Full list of author information is available at the end of the article
}

parasites infecting humans coexist and mixed species infections are common [1,2]. For improving accuracy in large epidemiological studies, molecular diagnostic tools permitting high through put analysis for the identification and quantification of malaria parasites would be of great benefit.

Traditionally, light microscopy (LM) examination of blood smears has been considered the gold standard for the diagnosis of malaria [3]. LM has clear advantages

\section{(Ciomed Central}


over molecular typing, since it incurs only low costs, does neither need complex sample preparation nor advanced technology and permits species identification and quantification [4]. However, the role of LM as a gold standard has been questioned due to false negative results at low parasitemia and frequent errors in species identification in mixed infections $[5,6]$.

The availability of commercial rapid diagnostic tests (RDT) has greatly facilitated the in situ diagnosis of malaria infections in the field. The advantages of RDT are that they do not need special preparation of the sample and diagnostic results become immediately available [7]. However, their use is limited due to lack of sensitivity for Plasmodium vivax, Plasmodium malariae and Plasmodium ovale [8].

Nested PCR (nPCR), first described by Snounou and co-workers [9], is a widely used method and is considered as a molecular gold standard due to its good performance in detection of mixed species infection. This assay amplifies the multicopy $18 \mathrm{~S}$ rRNA genes of the four Plasmodium species infecting humans. Even though this genotyping technique is now performed in many field laboratories in endemic countries, its use for routine clinical diagnosis is limited, because the analysis is time consuming due to the need of multiple reactions per sample, and the risk of contamination through the requirement for $\mathrm{nPCR}$ [9]. Moreover, the technique is not quantitative.

In recent years, various real time quantitative PCR (qPCR) assays have been developed for the detection of Plasmodium species, with most assays targeting the $18 \mathrm{~S}$ rRNA genes. Two of these methods detect the genus Plasmodium using generic primers, and thus do not distinguish between species $[10,11]$. Sybergreen reagent has been used by other groups, to identify the four Plasmodium species infecting humans in a single reaction by melting curve analysis $[8,12]$. The use of TaqMan probes contributed an additional level of specificity to qPCR assays [13]. However, using a single pair of primers for Plasmodium genus detection in duplex assays, introduces competition for amplification among species, which likely leads to difficulties in detecting mixed infections [14]. In order to address this issue, various groups had used different strategies [15-18]. The multiplex PCR-ligase detection reaction-fluorescent microsphere assay (PCR_LDR_FMA) has also been used in molecular epidemiological studies for simultaneously detecting all four Plasmodium species [19].

Despite the variety of molecular tools available for the diagnosis of malaria and their wide use for the diagnosis of imported malaria in travel clinics, implementation of these techniques in endemic areas has remained limited until now. Even though the low sensitivity and limited detection of asymptomatic and mixed infections by LM constitutes a critical shortfall for some epidemiological studies, LM remains to date the most frequently used method for the diagnosis of malaria in endemic areas. The transfer of molecular techniques for diagnosis of malaria to laboratories in endemic settings is essential for overcoming the limitations by LM. Moreover, a molecular technique with quantification capacity contributes to correctly estimate the burden of Plasmodium species often found in concomitant infections and will be a valuable tool to explore competition in mixed infections.

A qPCR assay initially developed for malaria diagnosis in returning travellers at a reference laboratory was implemented and validated at the PNG Institute of Medical Research (IMR). This assay was chosen because it detects with high specificity all four Plasmodium species which jointly occur in our study area in PNG. The performance of this qPCR assay in conditions of a field laboratory and on field samples was compared to light microscopy, nPCR and PCR_LDR_FMA results.

\section{Methods}

\section{Study site and sample collection}

Samples were collected in 10 villages from Ilahita area of East Sepik Province, PNG [2], in the framework of a longitudinal cohort study conducted during 2008. As part of the baseline, venous blood samples were obtained from 452 children one to five years of age, after written informed consent was obtained from parents or legal guardians of each child. This genotyping study was approved by the PNG IMR Institutional Review Board (IMR IRB 0720) and PNG Medical Research Advisory Committee (MRAC 07.34).

\section{Blood smear examination}

Thick/thin blood smears were prepared as described previously $[20,21]$. Blood smears were stained with a $5 \%$ Giemsa solution and examined independently by two microscopists, with a third microscopist reading for those slides with discrepant results. A minimum of 200 microscopic fields were examined at a magnification of $1000 \times$ using oil immersion optics before a slide was declared negative for malaria parasites by LM. Plasmodium species were quantified by counting infected parasites over 200 leukocytes. Conversion of parasite counts into parasites/ $\mu \mathrm{L}$ was performed assuming a mean leukocyte count of $8000 / \mu \mathrm{L}$ whole blood. Routinely slides were read twice. Discordant results were evaluated by a third slide reading. Final species diagnosis was based on the majority agreement.

\section{DNA template extraction and amplification}

DNA was extracted from $200 \mu \mathrm{L}$ whole blood (venous blood collected in EDTA anti-coagulant) using QIAamp 
96 DNA Blood Mini Kit (QIAGEN, Valencia, CA), and eluted in a final volume of $200 \mu \mathrm{L} \mathrm{dH} 2 \mathrm{O}$ according to the supplier's instructions. nPCR was carried out as described [22].

PCR_LDR_FMA was carried out as described elsewhere [19]. Mixes of serially diluted plasmids containing inserts of $P$. falciparum, $P$. vivax, $P$. malariae or $P$. ovale $18 \mathrm{~S}$ rDNA were used as positive controls in addition to $P$. falciparum or $P$. vivax positive samples obtained from field isolates. The threshold for positivity for each species was determined using the mean value obtained from negative controls for each species, plus three times the standard deviation.

The primers and probes of the qPCR assay are listed in Table 1 . In the design of a duplex qPCR, the probes combined in one reaction carried different fluorescent labels at their 5' ends. All four probes carried a black hole quencher (BHQ) at their 3'ends. The analytical specificity of primers and probes were evaluated for each Plasmodium species in silico by Blast searches and experimentally by using gDNA of the three alternatives Plasmodium species or of related blood borne parasites. To minimize costs of consumables, duplex reactions were performed in a final volume of 12.5 $\mu \mathrm{L}$. Amplification and detection of the amplified product was performed in an iQcycling BioRad system, using iQSupermix from BioRad. The P. falciparum/P. vivax (Pf/Pv) duplex reaction contained $2.5 \mu \mathrm{L}$ DNA (corresponding to $2.5 \mu \mathrm{L}$ whole blood), $6.25 \mu \mathrm{L}$ SuperMix iQ (BioRad), 0.35 $\mu \mathrm{L} P f$ primer mix $(50 \mu \mathrm{M}), 0.35 \mu \mathrm{L}$ of $P v$ primer mix 50 $\mu \mathrm{M}, 0.375 \mu \mathrm{L}$ of $P f$ probe $(10 \mu \mathrm{M}), 0.375 \mu \mathrm{L}$ of $P v$ probe $(10 \mu \mathrm{M})$ and $2.3 \mu \mathrm{L}$ double distilled water. The $P$. malariae/P. ovale $(\mathrm{Pm} / \mathrm{Po})$ duplex reaction contained equivalent amounts and concentrations of the respective primers and probes. The thermal profile used was 2 minutes at $50^{\circ} \mathrm{C}$, followed by 10 minutes at $95^{\circ} \mathrm{C}$ and 45 cycles of 15 seconds at $95^{\circ} \mathrm{C}$ and 1 minute at $58^{\circ} \mathrm{C}$.

\section{qPCR validation}

Evaluation of PCR efficiency and reproducibility was performed on standard curves using four positive control plasmids with the respective amplicons inserted. Geometric mean and standard deviation were calculated from triplicates in three independent assays. Standard curve for each Plasmodium species was made from a 10 -fold serial dilution of the control plasmids ranging from $10^{6}$ copies $/ \mu \mathrm{L}$ to 10 copies $/ \mu \mathrm{L}$.

Amplification efficiencies for the different primer pairs and probes were calculated with the formula:

Efficiency $=10^{(-1 / \text { Slope })}-1$. Inter-assay coefficients of variation $(\mathrm{CVs})$ were calculated for each plasmid dilution separately as $(\mathrm{SD} /$ mean)*100 using the $\mathrm{Ct}$ values from different runs.

Reproducibility of qPCR was further analysed by repeating $10 \%$ of all 452 field-samples in the same laboratory, but at different time points. External quality assurance was performed in addition.

The amount of target in an unknown sample was quantified by converting the threshold cycle $(\mathrm{Ct})$ into template copy number by using the four standard curves. Samples yielding $\mathrm{Ct}$ values equal or higher than 40 were considered Plasmodium species negative [23].

\section{Statistical analysis}

Results from qPCR was compared with those from LM, nPCR and PCR-LDR-FMA. Agreement between diagnostic methods was determined by calculating Kappa Statistics with $95 \%$ confidence intervals. Values were interpreted with the Landis and Koch classification [24] as follows: $\mathrm{k}=0.41-0.60$, moderate agreement, $\mathrm{k}=0.61$ 0.80 , good agreement; $\mathrm{k}=0.81-1.00$, almost perfect agreement beyond chance. Prevalence for each species was calculated by dividing the number of positive samples through by the number of all samples tested and

Table 1 Primers and probes for qPCR

\begin{tabular}{|c|c|c|c|}
\hline Species & $\begin{array}{c}\text { Primer/ } \\
\text { probe }\end{array}$ & Sequence $5^{\prime}-3^{\prime}$ & Fluorescent label \\
\hline P. falciparum & Fal_F & TATTGCTITTGAGAGGTTITGTTACTTTG & \\
\hline P. falciparum & Fal_R & ACCTCTGACATCTGAATACGAATGC & \\
\hline P. falciparum & Fal_P & ACGGGTAGTCATGATTGAGTT & FAM-BHQ \\
\hline P. vivax & Viv_F & GCTITGTAATTGGAATGATGGGAAT & \\
\hline P. vivax & Viv_R & ATGCGCACAAAGTCGATACGAAG & \\
\hline P. vivax & Viv_P & AGCAACGCTTCTAGCTTA & HEX-BHQ \\
\hline P.malariae & Mal_F & TGCCGACTAGGTGTTGGATGAT & \\
\hline P.malariae & Mal_R & CTAGTGAGTTTCCCCGTGTTGAGT & \\
\hline P.malariae & Mal_P & TGTTTCTTTAGATAGCTTCCTTCAG & FAM-BHQ \\
\hline P. ovale & Ova_F & CCAGCTCCAATAGCGTATATTAAA & \\
\hline P. ovale & Ova_R & ACACATTTTGSATAAGGAATGCAAAG* & \\
\hline P. ovale & Ova_P & TATAAGATGCTTAGRCAATACAACGTATCTG* & HEX-BHQ \\
\hline
\end{tabular}

*Oligo includes a wobble (S: G or C; R: A or G) 
McNemar test was calculated in order to test for significance. $P$ values $<0.05$ were considered statistically significant. Correlation between LM and qPCR quantification was calculated by pairwise correlation coefficient. All statistical calculations were performed with STATAv.10 statistical software.

\section{Results}

\section{Optimization of qPCR assay}

The large scale of field work required optimization of molecular testing with the aim to reduce costs. Two strategies were applied: multiplexing the qPCR and reducing the reaction volume. The performance of the two duplex qPCRs were evaluated by analysing dilution series of the two respective control plasmids in various ratios (Additional file 1). These experiments demonstrated very little inhibition when 200 templates and above were present in the reaction. Only the detection of minute template concentrations, as low as 20 templates per reaction, was slightly inhibited by excess amounts of the alternative template, i.e. in a 100 times excess.

Table 2 shows the duplex qPCR efficiencies for all four templates after reducing the reaction volume to $12.5 \mu \mathrm{L}$. The dilution series was performed with control plasmids containing inserts corresponding to the Plasmodium species-specific PCR product. Similar slopes were observed for all species, demonstrating similar amplification efficiency throughout the tested range and therefore comparability between quantification results. Correlation coefficients around 0.980 demonstrated that our assays were linear over the entire quantification range. The inter-assay coefficients of variation (CV) between $0.3 \%$ and $5 \%$, obtained in all six dilutions, indicated reproducible results (Additional file 2).

Reproducibility of our assay was investigated by reanalysing $10 \%$ of the 452 samples in the same laboratory. In $8 / 45$ compared samples discordant results were recorded. Four of these discrepancies occurred in cases of low density in the range of 10-100 copies/ $\mu \mathrm{L}$. Ct values were between 37 and 39 in the positive ones of the duplicate tests. Two other discrepancies were observed in mixed species infections, whereby the minority species was detected in only one of the duplicate experiments in the range of 10 to 100 copies $/ \mu \mathrm{L}$.

Table 2 qPCR Efficiencies for each Plasmodium species

\begin{tabular}{ccccc}
\hline & Slope & Efficiency $\%$ & Interception & R squared \\
\hline P. falciparum & -3.50 & $93.17 \%$ & 39.33 & 0.987 \\
P. vivax & -3.59 & $89.7 \%$ & 38.56 & 0.975 \\
P. malariae & -3.57 & $90.46 \%$ & 40.26 & 0.989 \\
P. ovale & -3.51 & $92.54 \%$ & 38.83 & 0.988 \\
\hline
\end{tabular}

\section{Comparison of diagnosis of Plasmodium infection by qPCR, nPCR, PCR-LDR and LM}

Prevalence values for $P$. falciparum showed significant differences when all detection techniques were considered $(40.9 \%$ by qPCR, $43.8 \%$ by nPCR and $47.1 \%$ by LDR, p-values < 0.05). However, greater agreement was observed for qPCR compared to LM $(\mathrm{k}=0.621)$ or to PCR-LDR ( $\mathrm{k}=0.750)$. Almost perfect agreement was obtained for prevalence by qPCR and by nPCR $(\mathrm{k}=$ 0.869) (Table 3). Prevalence for P. vivax by qPCR was not significantly different from that by LDR (65.7\% versus $67.5 \%$, p-value > 0.5), but a statistically significant difference was seen when comparing to nPCR (prevalence $73.2 \%$, p-value $<0.05$ ) or LM (prevalence 54.9\%, p-value $<0.05)$. Overall, $P$. vivax prevalence by qPCR was in moderate agreement with the other assays $(0.596$ with nPCR, 0.553 with PCR-LDR and 0.530 with LM). Prevalence values for $P$. malariae and $P$. ovale were highly discrepant between the different techniques. Prevalence of $P$. malariae by qPCR (4.7\%) differed significantly from that of PCR-LDR (prevalence $7.7 \%$, p-value $<0.05$ ), and from that of nPCR (prevalence 3.3\%, $\mathrm{p}$-value $<0.05)$. Moreover, prevalence of $P$. ovale was higher by qPCR (7.3\%) compared to that of LDR (4.4\%) and $\mathrm{nPCR}(3.8 \%)$. The difference between all these values was statistically significant. Prevalence by LM was significantly lower for all four species $(25.4 \%$ for P. falciparum, $54.9 \%$ for $P$. vivax, $2.4 \%$ for $P$. malariae and $0 \%$ for $P$. ovale, $\mathrm{p}$-values $<0.05)$. $P$. malariae and $P$. ovale moderate kappa scores were retrieved when comparing qPCR with the other assays (Table 4).

\section{Quantification of Plasmodium parasites}

For P. falciparum, quantification by qPCR correlated well with microscopy counts when both were positive $\left(\mathrm{R}^{2}=0.8253\right)$. A substantial number of samples were negative for $P$. falciparum by LM, but positive by qPCR; in the scatter blot in Figure 1A these negative samples locate to the ordinate. Very few samples were LM positive, but qPCR negative for P. falciparum. As the densities of these samples were not extremely low and all of

Table 3 P. falciparum versus P. vivax agreement among different diagnostic techniques

\begin{tabular}{|c|c|c|c|c|}
\hline & qPCR & $\mathrm{nPCR}$ & PCR-LDR & LM \\
\hline qPCR & & $\begin{array}{c}0.869 \\
\text { perfect }\end{array}$ & $\begin{array}{c}0.750 \\
\text { substantial }\end{array}$ & $\begin{array}{c}0.621 \\
\text { substantial }\end{array}$ \\
\hline $\mathrm{nPCR}$ & $\begin{array}{c}0.596 \\
\text { moderate }\end{array}$ & & $\begin{array}{c}0.737 \\
\text { substantial }\end{array}$ & $\begin{array}{c}0.562 \\
\text { moderate }\end{array}$ \\
\hline $\begin{array}{l}\text { PCR- } \\
\text { LDR }\end{array}$ & $\begin{array}{c}0.553 \\
\text { moderatee }\end{array}$ & $\begin{array}{c}0.609 \\
\text { substantial }\end{array}$ & & $\begin{array}{c}0.517 \\
\text { moderate }\end{array}$ \\
\hline LM & $\begin{array}{c}0.530 \\
\text { moderate }\end{array}$ & $\begin{array}{c}0.513 \\
\text { moderate }\end{array}$ & $\begin{array}{c}0.611 \\
\text { substantial }\end{array}$ & \\
\hline
\end{tabular}

P. falciparum (normal case). P. vivax (italics). 
Table 4 P. malariae versus P. ovale agreement among different diagnostic techniques

\begin{tabular}{|c|c|c|c|c|}
\hline & qPCR & nPCR & PCR-LDR & LM \\
\hline qPCR & & $\begin{array}{c}0.595 \\
\text { moderate }\end{array}$ & $\begin{array}{c}0.545 \\
\text { moderate }\end{array}$ & $\begin{array}{c}0.484 \\
\text { moderate }\end{array}$ \\
\hline nPCR & $\begin{array}{c}0.621 \\
\text { substantial }\end{array}$ & & $\begin{array}{c}0.455 \\
\text { moderate }\end{array}$ & $\begin{array}{c}0.446 \\
\text { moderate }\end{array}$ \\
\hline PCR-LDR & $\begin{array}{c}0.541 \\
\text { moderate }\end{array}$ & $\begin{array}{c}0.577 \\
\text { moderate }\end{array}$ & & $\begin{array}{c}0.458 \\
\text { moderate }\end{array}$ \\
\hline
\end{tabular}

P. malariae (normal case). P. ovale (italics).

them were co-infected with other Plasmodium species, microscopic misclassification of the Plasmodium species is likely. The correlation between microscopy counts and qPCR-based densities was lower for P. vivax $\left(\mathrm{R}^{2}=\right.$ 0.5049). This lower agreement might be due to overall lower densities, possibly around the detection limit, in $P$. vivax infections. Such stochastic variation in parasite presence likely was responsible for negative slide results as well as for a negative qPCR, both indicated by numerous data points on the coordinates in Figure 1B. Generally in all PCR-based techniques low densities lead to alternating positive or negative results in repeated experiments due to the coincidental lack of any template in some reactions. Furthermore, mixed species infections, where $P$. vivax is found at a very low density, contribute to a lower correlation between microscopy count and qPCR-based densities likely due to misclassification by LM. To illustrate this, $P$. vivax correlation coefficient significantly increased up to $R^{2}=0.7220$ when only single infections by qPCR are analysed, but was as low as $R^{2}=0.4372$ when only mixed infections are considered. Few of the samples positive for $P$. malariae by qPCR and none of the $P$. ovale positive samples were identified by light microscopy.

\section{Discussion}

In preparation of major molecular epidemiological field studies in PNG essential parasite detection techniques were compared under conditions of a laboratory close to the field site and located in a malaria endemic country. The diagnostic requirements were: (i) good performance in the detection of mixed species infections, as all four species concurrently occur in PNG, (ii) recognition of $P$. malariae and $P$. ovale variants present in the study area, (iii) high through put capacity and robustness of assay, (iv) quantitative results and (v) reasonable costs. The qPCR assay described here was implemented and validated at the PNG-IMR site in Madang, demonstrating the feasibility of applying state of the art techniques in this context. In the meantime the qPCR assay is routinely implemented for molecular diagnosis in large scale epidemiologic studies at IMR.

As part of test validation in the field, the performance of this qPCR assay for Plasmodium species discrimination was compared to two other PCR-based assays (nPCR and LDR) and to LM. Traditionally, test outcomes for different assays are compared to an established 'gold-standard' in order to calculate sensitivity and specificity estimates and to evaluate the performance of newly developed tests. The classical 'gold standard' for malaria diagnosis has been LM [3], however, with the appearance of new molecular diagnostic tools, LM has become less suitable for this purpose due to its lower sensitivity than molecular methods [6]. Even though the nPCR developed by Snounou et al [9] has been extensively used as 'gold-standard' for molecular
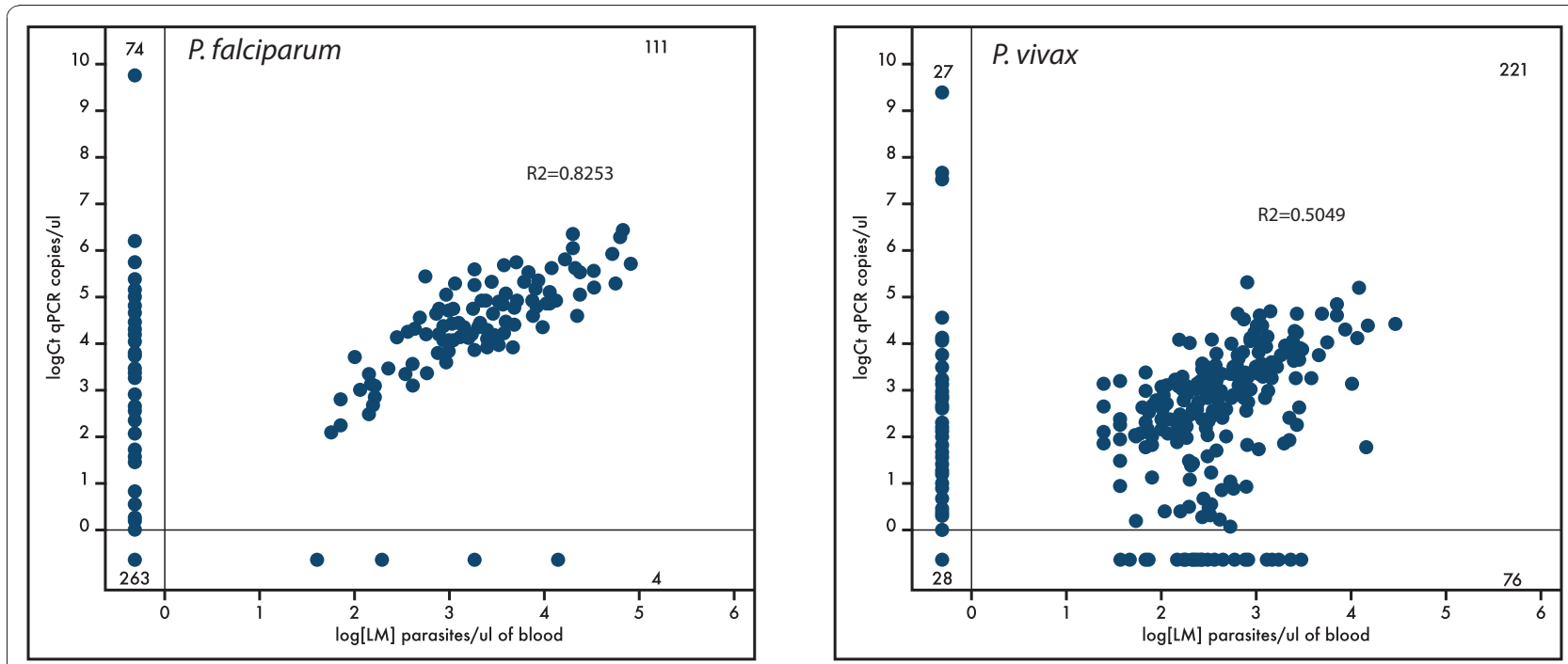

Figure 1 Comparison of quantification assessed by light microscopy versus qPCR. 
diagnosis $[25,26]$, the concept of using a 'gold-standard' for the evaluation of new assays is being questioned by various authors, which alternatively propose the use of 'non-gold standard' approaches [27,28].

The agreement between qPCR and the other techniques was substantial for $P$. falciparum, but only moderate for $P$. vivax, $P$. malariae and $P$. ovale. In particular, the agreement between $\mathrm{qPCR}$ and $\mathrm{nPCR}$ for $P$. falciparum detection was almost perfect. The lower agreement between PCR-LDR and nPCR, together with the higher prevalence shown by PCR-LDR (47.1\% compared to $40.9 \%$ by qPCR and $43.8 \%$ by nPCR), may indicate false positive results by LDR. This is supported by our pairwise analysis and the agreement of two independent PCR based assays, namely nPCR and qPCR. However, in absence of a suitable diagnostic 'gold standard', it remains unclear if those 33 samples positive for LDR but negative by the two alternative molecular methods, represent a greater sensitivity of LDR or simply false positives. This issue cannot be easily resolved in a study involving 'unknown' samples from the field, potentially infected by four different Plasmodium species.

$P$. vivax prevalence was higher by $\mathrm{nPCR}$ than by both, qPCR and PCR-LDR (73.2\% by nPCR, $65.7 \%$ by qPCR and $67.5 \%$ by PCR-LDR). This again could reflect false postitives by nPCR or lower sensitivity by both other molecular methods. Our observations in GPCR validation using plasmid template suggested that qPCR of $P$. vivax is lightly compromised by performing a duplex $P f / P v$ reaction. nPCR involves a very high number of cycles ( 55 cycles by nPCR versus 45 cycles by qPCR and 35 cycles by LDR), and therefore is expected to show maximal sensitivity. Despite measurements taken over 45 cycles in qPCR, we followed the consensus rule for considering a sample positive, i.e. a Ct value $<40$ [23]. In our samples this led to the loss of 9 samples with $\mathrm{Ct}$ values for $P$. vivax between 40 and 43.6 cycles, which otherwise would have increased the sensitivity of the assay. Further analysis was performed on samples with discrepant results for $P$. vivax ( $P$. vivax negative samples by qPCR and positive by nPCR). Most of these samples were mixed infections by nPCR and harboured P. falciparum with more than 10,000 copies/ $\mu$ l. Thus competition for amplification at the beginning of the PCR due to $P$. falciparum high densities may be precluding P. vivax detection [14]. 14/16 of the remaining samples were also negative by LM. Most likely these very lowgrade $P$. vivax infections were missed. The scarcity of the template in case of a very low parasite density is expected to lead to imperfect detection. Prevalence for $P$. malariae and $P$. ovale were low with significant differences between assays, even though the agreement between pairwise compaired methods was moderate. Higher prevalence for $P$. malariae detection by LDR is likely to occur as a result of false positive results, probably occurring due to high background noise of the $P$. malariae probe used in the assay. Low detection of $P$. ovale by $\mathrm{nPCR}(3.8 \%)$ is due to the use of a primer pair with sub-optimal amplification of $P$. ovale sequences present in the study area. Finally, LM measured the lowest prevalence for all four Plasmodium species.

The major advantage of qPCR over the other compared molecular techniques was the quantification of parasite densities. Parasite densities shown as copies of $18 \mathrm{~S}$ rRNA template/ $\mu \mathrm{L}$ were quantified by converting the threshold cycle $(\mathrm{Ct})$ into template copy number by using the standard curves. When correlating quantification by qPCR with LM counts in samples where both techniques showed positive results, a high correlation for $P$. falciparum $\left(R^{2}=0.8253\right)$ and a lower correlation for $P$. vivax $\left(\mathrm{R}^{2}=0.5049\right)$ was found. But for $P$. vivax this correlation of parasite densities by qPCR and LM increased when only single infections were taken into account. Therefore, our results suggest a variation in the detection limit in both methods, due to overlooking $P$. vivax in case of an overwhelming $P$. falciparum infection. Difficulties in identifying $P$. vivax by LM arise when this parasite is found at low densities and in mixed infections. The high $P$. falciparum densities found in the samples identified as mixed infection by qPCR (> 10000 target copies/ $\mu$ l) further supports this explanation. The correlation for $P$. malariae and $P$. ovale could not be analysed due to poor detection of both species by LM.

The qPCR assay was found optimal for both tasks, detection of all four Plasmodium species and quantification. The latter could only be analyzed for $P$. falciparum and $P$. vivax. Overall qPCR shows substantial agreement with other molecular techniques for the detecting prevalence of $P$. falciparum and $P$. vivax, while moderate agreement was observed for $P$. malariae and $P$. ovale. It is clear, that sensitivity of our qPCR assay can be increased by simply performing independent reactions of each Plasmodium species. However, this would substantially increase costs. Limiting factors, such as duplex assays, need to be balanced against costs or work load. The specific research objectives of a particular study should guide the choice of experimental procedures.

Overall, the superior performance of PCR based methodologies over LM has been clearly demonstrated by these results and others. In a recent study conducted in Benin, a high number of children (between $27 \%$ and $44 \%$ ) aged 5 or above, who initially had negative RDT tests (most also with negative blood slides), were later found to be infected with P. falciparum using PCR [29]. These undetected submicroscopic infections have an enormous impact for malaria transmission in endemic areas. In a time where malaria erradication has become 
the primary goal of malaria agendas, the accurate estimation of the burden of malaria infection is imperative to control transmission.

\section{Conclusions}

In conclusion, this qPCR assay was sensitive and specific for the detection of all four Plasmodium species and results agreed well with other molecular techniques tested. Added advantage of the qPCR assay is quantification of parasite densities and a less laborious workflow. Moreover, the assay performed well in field samples and due to its high through put capacity, it is suitable for large scale epidemiological studies. Finally, a quantitative assay is of a greatest value for monitoring of malaria control programmes.

\section{Additional material}

Additional file 1: Table S1. Effect of mixed-species infection on sensitivity of duplex qPCR.

Additional file 2: Table S2. Inter-assay reproducibility of qPCR assay.

\section{Acknowledgements}

We are particularly thankful to all the children of Ilahita area, their parents and/or guardians who participated in the study. We thank the staff from the Institute of Medical Research in Maprik for their assistance in collecting and processing blood samples, to the staff from the Institute of Medical Research in Madang for malaria microscopy readings and to Nomin-Dora Tenakanai for her help with the Bioplex analysis. This investigation received financial support from the Fundación Privada CELLEX (Catalonia, Spain).

\section{Author details}

${ }^{1}$ Papua New Guinea Insitute of Medical Research, Madang 511, Papua New Guinea. ${ }^{2}$ Barcelona Centre for International Health Research (CRESIB), Hospital Clinic/IDIBAPS, Universitat de Barcelona; Roselló 132, 4a planta, 08036, Barcelona, Spain. ${ }^{3}$ Swiss Tropical and Public Health Institute, Socinstrasse 57, CH-4002 Basel, Switzerland. ${ }^{4}$ University of Basel, Petersplatz 1, CH-4003 Basel, Switzerland. ${ }^{5}$ Center for Global Health and Diseases, Case Western Reserve University School of Medicine, Cleveland, Ohio. ${ }^{6}$ Institució Catalana de Recerca i Estudis Avançats (ICREA), Barcelona, Spain.

\section{Authors' contributions}

DM and IF conceived and designed QPCR assay and AR coordinated the study. DM validated $\mathrm{QPCR}$ in the reference lab and $A R$ in the endemic seating. IB and AR participated in the sample collection. JI did the DNA extractions from the blood samples. CB performed the PCR-LDR-FMA. AR performed $\mathrm{GPCR}$ and $\mathrm{nPCR}$ methods, statistical analysis and interpretation of the data. AR, IF and IM draft the manuscript. PZ and HP critically reviewed the manuscript. All authors have read and approved the final manuscript.

\section{Competing interests}

The authors declare that they have no competing interests.

Received: 14 September 2010 Accepted: 14 December 2010 Published: 14 December 2010

\section{References}

1. Mehlotra RK, Kasehagen LJ, Baisor M, Lorry K, Kazura JW, Bockarie MJ, Zimmerman PA: Malaria infections are randomly distributed in diverse holoendemic areas of Papua New Guinea. Am J Trop Med Hyg 2002, 67:555-562.
2. Mueller I, Galinski MR, Baird JK, Carlton JM, Kochar DK, Alonso PL, del Portillo HA: Key gaps in the knowledge of Plasmodium vivax, a neglected human malaria parasite. Lancet Infect Dis 2009, 9:555-566.

3. Organization WH: World malaria report 2008. Geneva, Switzerland: WHO Press; 2008.

4. Moody AH, Chiodini PL: Non-microscopic method for malaria diagnosis using OptiMAL IT, a second-generation dipstick for malaria pLDH antigen detection. Br J Biomed Sci 2002, 59:228-231.

5. Coleman RE, Sattabongkot J, Promstaporm S, Maneechai N, Tippayachai B, Kengluecha A, Rachapaew N, Zollner G, Miller RS, Vaughan JA, Thimasarn K, Khuntirat B: Comparison of PCR and microscopy for the detection of asymptomatic malaria in a Plasmodium falciparum/vivax endemic area in Thailand. Malar J 2006, 5:121.

6. Ochola LB, Vounatsou P, Smith T, Mabaso ML, Newton CR: The reliability of diagnostic techniques in the diagnosis and management of malaria in the absence of a gold standard. Lancet Infect Dis 2006, 6:582-588.

7. WHO: Malaria rapid diagnostic test performance: results of WHO product testing of malaria RDTs: round 1 (2008). Geneva: World Health Organization; 2009.

8. de Monbrison F, Angei C, Staal A, Kaiser K, Picot S: Simultaneous identification of the four human Plasmodium species and quantification of Plasmodium DNA load in human blood by real-time polymerase chain reaction. Trans $R$ Soc Trop Med Hyg 2003, 97:387-390.

9. Rubio JM, Benito A, Roche J, Berzosa PJ, Garcia ML, Mico M, Edu M, Alvar J: Semi-nested, multiplex polymerase chain reaction for detection of human malaria parasites and evidence of Plasmodium vivax infection in Equatorial Guinea. Am J Trop Med Hyg 1999, 60:183-187.

10. Lee MA, Tan CH, Aw LT, Tang CS, Singh M, Lee SH, Chia HP, Yap EP: Realtime fluorescence-based PCR for detection of malaria parasites. $J$ Clin Microbiol 2002, 40:4343-4345.

11. Farcas GA, Zhong KJ, Mazzulli T, Kain KC: Evaluation of the RealArt Malaria LC real-time PCR assay for malaria diagnosis. J Clin Microbiol 2004, 42:636-638.

12. Mangold KA, Manson RU, Koay ES, Stephens L, Regner M, Thomson RB Jr, Peterson LR, Kaul KL: Real-time PCR for detection and identification of Plasmodium spp. J Clin Microbiol 2005, 43:2435-2440.

13. Rougemont M, Van Saanen M, Sahli R, Hinrikson HP, Bille J, Jaton K: Detection of four Plasmodium species in blood from humans by $18 \mathrm{~S}$ rRNA gene subunit-based and species-specific real-time PCR assays. $J$ Clin Microbiol 2004, 42:5636-5643.

14. Bialasiewicz S, Whiley DM, Nissen MD, Sloots TP: Impact of competitive inhibition and sequence variation upon the sensitivity of malaria PCR. Clin Microbiol 2007, 45:1621-1623.

15. Perandin F, Manca N, Calderaro A, Piccolo G, Galati L, Ricci L, Medici MC, Arcangeletti MC, Snounou G, Dettori G, et al: Development of a real-time PCR assay for detection of Plasmodium falciparum, Plasmodium vivax, and Plasmodium ovale for routine clinical diagnosis. J Clin Microbiol 2004, 42:1214-1219.

16. Shokoples SE, Ndao M, Kowalewska-Grochowska K, Yanow SK: Multiplexed real-time PCR assay for discrimination of Plasmodium species with improved sensitivity for mixed infections. J Clin Microbiol 2009, 47:975-980.

17. Dormond L, Jaton-Ogay K, de Valliere S, Genton B, Bille J, Greub G: Multiplex real-time PCR for the diagnosis of malaria: correlation with microscopy. Clin Microbiol Infect 2010.

18. Vo TK, Bigot P, Gazin P, Sinou V, De Pina JJ, Huynh DC, Fumoux F, Parzy D: Evaluation of a real-time PCR assay for malaria diagnosis in patients from Vietnam and in returned travellers. Trans R SoC Trop Med Hyg 2007 101:422-428

19. McNamara DT, Kasehagen LJ, Grimberg BT, Cole-Tobian J, Collins WE, Zimmerman PA: Diagnosing infection levels of four human malaria parasite species by a polymerase chain reaction/ligase detection reaction fluorescent microsphere-based assay. Am J Trop Med Hyg 2006, 74:413-421.

20. Genton B, al-Yaman F, Beck HP, Hii J, Mellor S, Rare L, Ginny M, Smith T, Alpers MP: The epidemiology of malaria in the Wosera area, East Sepik Province, Papua New Guinea, in preparation for vaccine trials. II. Mortality and morbidity. Ann Trop Med Parasitol 1995, 89:377-390.

21. al-Yaman F, Genton B, Kramer KJ, Taraika J, Chang SP, Hui GS, Alpers MP: Acquired antibody levels to Plasmodium falciparum merozoite surface 
antigen 1 in residents of a highly endemic area of Papua New Guinea. Trans R Soc Trop Med Hyg 1995, 89:555-559.

22. Snounou G, Viriyakosol S, Jarra W, Thaithong S, Brown KN: Identification of the four human malaria parasite species in field samples by the polymerase chain reaction and detection of a high prevalence of mixed infections. Mol Biochem Parasitol 1993, 58:283-292.

23. Bustin SA: Why the need for qPCR publication guidelines?-The case for MIQE. Methods 50:217-226.

24. Landis JR, Koch GG: An application of hierarchical kappa-type statistics in the assessment of majority agreement among multiple observers. Biometrics 1977, 33:363-374

25. Mens P, Spieker N, Omar S, Heijnen M, Schallig H, Kager PA: Is molecular biology the best alternative for diagnosis of malaria to microscopy? A comparison between microscopy, antigen detection and molecular tests in rural Kenya and urban Tanzania. Trop Med Int Health 2007, 12:238-244.

26. Mixson-Hayden T, Lucchi NW, Udhayakumar V: Evaluation of three PCRbased diagnostic assays for detecting mixed Plasmodium infection. BMC Res Notes 2010, 3:88.

27. Koukounari A, Webster JP, Donnelly CA, Bray BC, Naples J, Bosompem K Shiff C: Sensitivities and specificities of diagnostic tests and infection prevalence of Schistosoma haematobium estimated from data on adults in villages northwest of Accra, Ghana. Am J Trop Med Hyg 2009, 80:435-441.

28. de Clare Bronsvoort BM, von Wissmann B, Fevre EM, Handel IG, Picozzi K, Welburn SC: No gold standard estimation of the sensitivity and specificity of two molecular diagnostic protocols for Trypanosoma brucei spp. in Western Kenya. PloS One 2010, 5:e8628.

29. Faucher JF, Aubouy A, Beheton T, Makoutode P, Abiou G, Doritchamou J, Houze $\mathrm{P}$, Ouendo E, Deloron $\mathrm{P}$, Cot M: What would PCR assessment change in the management of fevers in a malaria endemic area? A school-based study in Benin in children with and without fever. Malar $J$ 2010, 9:224.

doi:10.1186/1475-2875-9-361

Cite this article as: Rosanas-Urgell et al:: Comparison of diagnostic methods for the detection and quantification of the four sympatric Plasmodium species in field samples from Papua New Guinea. Malaria Journal 2010 9:361.

\section{Submit your next manuscript to BioMed Central and take full advantage of:}

- Convenient online submission

- Thorough peer review

- No space constraints or color figure charges

- Immediate publication on acceptance

- Inclusion in PubMed, CAS, Scopus and Google Scholar

- Research which is freely available for redistribution

Submit your manuscript at www.biomedcentral.com/submit
Biomed Central 